Ann. Sci. forest., 1974, 31 (4), 229-248.

\title{
UTILISATION DES PHOTOGRAPHIES HÉMISPHÉRIQUES POUR LE CUBAGE DES ARBRES SUR PIED
}

\author{
M. DUCREY et M. BARTOLI \\ avec la collaboration technique de J. Aubert et M. Pitsch \\ Station de Sylviculture et de Production \\ Centre national de Recherches forestières, I.N.R.A., \\ Champenoux 54280 Seichamps
}

\begin{abstract}
RÉSUMÉ
L'auteur propose l'utilisation d'un nouveau « dendromètre » pour cuber les arbres sur pied. Il s'agit d'un appareil photographique muni d'un objectif fish-eye de $180^{\circ}$ d'angle de visée. Les hauteurs et les diamètres sont calculés à partir des mesures faites sur les agrandissements photographiques. La précision de cette méthode ( 5 p. 100 sur le volume total d'un arbre) est voisine de celle obtenue par les autres appareils permettant le cubage par billons successifs des arbres sur pied.
\end{abstract}

L'estimation du volume des arbres sur pied est un problème toujours préoccupant bien que de très nombreuses méthodes et des appareils très divers aient été utilisés pour résoudre cette question.

Le « dendromètre » que nous présentons dans cet article n'est absolument pas spécifique de ce problème. Il s'agit d'un appareil photographique équipé d'un objectif « fisheye » (œil de poisson) qui prend des photographies circulaires dites photographies hémisphériques. Nous utilisons actuellement ce type de photographies pour l'étude du microclimat lumineux en forêt (DuCREY, 1970, 1971). Mais elles peuvent avoir de très nombreuses autres utilisations, dans l'architecture ou la météorologie par exemple.

L'objectif « fish-eye » est un grand angulaire de $180^{\circ}$ très exactement, qui donne de tout ce qui est situé au-dessus d'un plan de référence, une image circulaire suivant les lois de la projection équidistante.

Dans le cas où l'appareil est disposé de telle manière que l'axe optique de l'objectif passe par le zénith, et si on assimile le ciel à une demi-sphère, tous les points situés à une 
même distance angulaire du zénith, sont placés sur un cercle dont le centre est l'image de ce zénith. Le rayon des cercles en question est d'autre part proportionnel à la distance angulaire entre le zénith et les points considérés. Toujours dans ces conditions d'utilisation, les images des droites verticales convergent au centre de la photographie. La photographie ci-contre, prise dans une peupleraie, illustre parfaitement ce phénomène.

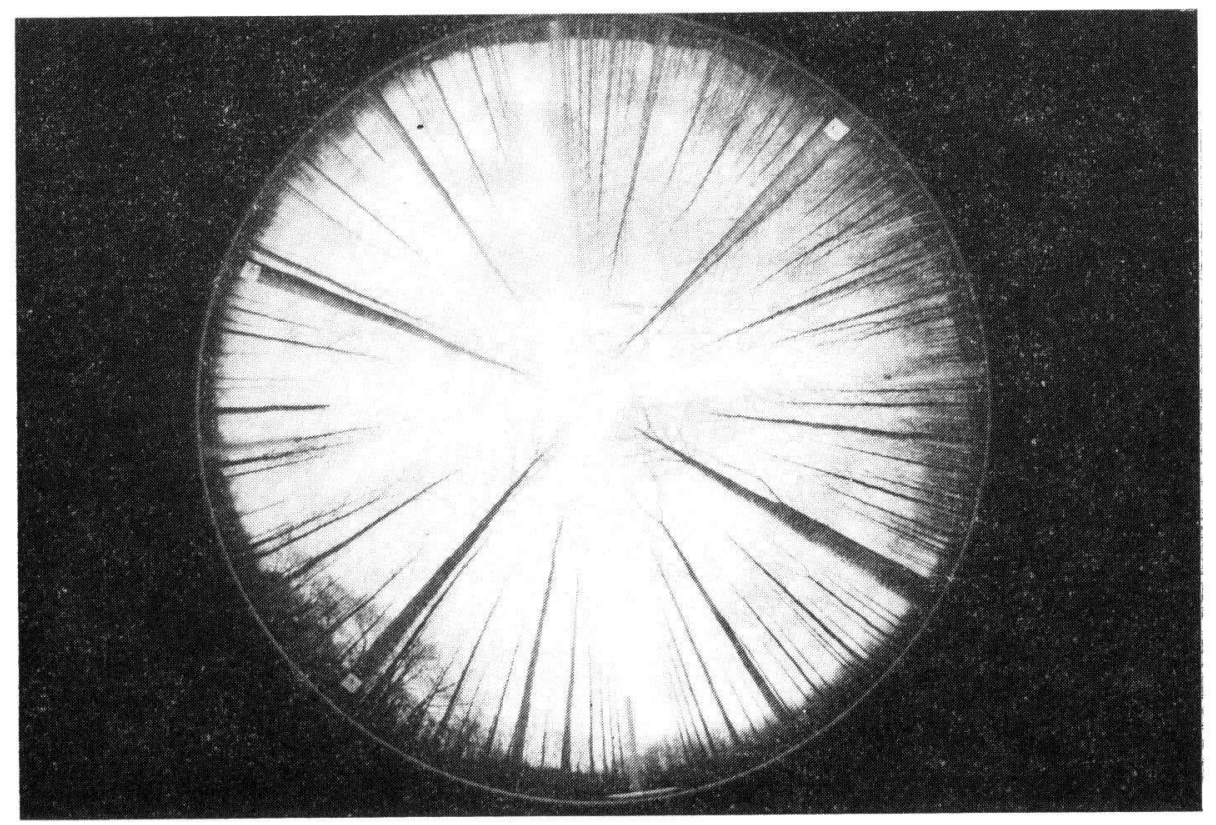

Рното. - Photographie hémisphérique d'une peupleraie en hiver Photograph. - Hemispherical photograph of a poplar stand in winter

Ces propriétés rendent possible, sur les photographies, le calcul de distances horizontales et verticales en conditions naturelles. En d'autres termes, on peut donc mesurer des hauteurs et des diamètres, c'est-à-dire « cuber » des arbres par billons successifs.

Nous développons cette idée nouvelle tout au long de cet article en commençant par décrire le principe de la méthode imaginée, puis en donnant quelques premières indications pratiques sur la manière de procéder, tant sur le terrain que pour le traitement des données au bureau.

Il reste bien entendu que nous n'en sommes encore, au plan des applications, qu'à la phase exploratoire que nous estimons déjà prometteuse.

\section{I. - PRINCIPE DE LA MÉTHODE UTILISÉE}

Nous allons, dans ce chapitre, démontrer un certain nombre de relations mathématiques qui permettent de passer, des mesures effectuées sur les photographies, aux dimensions réelles des arbres. 


\section{1. - Calcul des hauteurs et des diamètres}

a) Cas d'un terrain horizontal.

Nous supposerons connue la distance $l$ entre l'appareil photographique et l'arbre. La mesure de $l$, qui est d'ailleurs, en dehors de la prise de vue, une des seules opérations à faire sur le terrain, devra être faite avec le maximum de précautions. C'est très exactement la distance entre le centre de l'arbre et l'axe optique de l'appareil photographique.

Nous nous proposons de calculer le diamètre de l'arbre au point B (figure 1) dont la hauteur angulaire au-dessus de l'horizon, comptée à partir de l'appareil photographique, est égale à $\beta$.

La hauteur réelle du point $\mathrm{B}$, au-dessus du plan de référence est :

$$
h_{\beta}=l \operatorname{tg} \beta \text {. }
$$

Si l'appareil photographique $\mathrm{P}$ est situé à une hauteur $h_{0}$ au-dessus du sol, le plan de référence coupe l'arbre au point A qui est aussi à la hauteur $h_{0}$.

La hauteur par rapport au sol du point de mesure B est alors :

$$
\mathrm{H}_{\beta}=h_{0}+l \operatorname{tg} \beta \text {. }
$$

En ce qui concerne le diamètre, la photographie fournit un diamètre apparent $d_{\beta}$ (figure 1) qui est l'intersection de l'image de l'arbre avec le cercle dont le rayon correspond à la hauteur angulaire $\beta$.

La figure 2 traduit de manière imagée le passage du diamètre réel $D_{\beta}$ pour la hauteur angulaire $\beta$, au diamètre apparent $d_{\beta}$ de la photographie. L'arbre est schématisé par un cône. On a aussi fait figurer la sphère de projection équidistante et enfin la représentation plane (photographie hémisphérique) de cette projection.

Le dièdre dont l'arête passe par la verticale de l'appareil photographique et dont les côtés sont tangents à la section dont on cherche le diamètre $\left(D_{\beta}\right)$, a un angle égal à $\alpha$.

D'où : $\alpha=\frac{\mathrm{D}_{\beta}}{l}$.

Dans le système de projection adopté, l'angle $\alpha$ est l'angle sous lequel est vue l'image $d_{\beta}$ de $\mathrm{D}_{\beta}$ depuis le centre de la photographie hémisphérique.

On a done :

$\alpha=\frac{d_{\beta}}{r_{\beta}}$ si $r_{\beta}$ est le rayon, sur la photographie, du cercle de hauteur angulaire $\beta$ et si $\alpha$ est petit, ce qui est toujours le cas.

Finalement $: \mathrm{D}_{\beta}=\frac{d_{\beta} l}{r_{\beta}}$. 


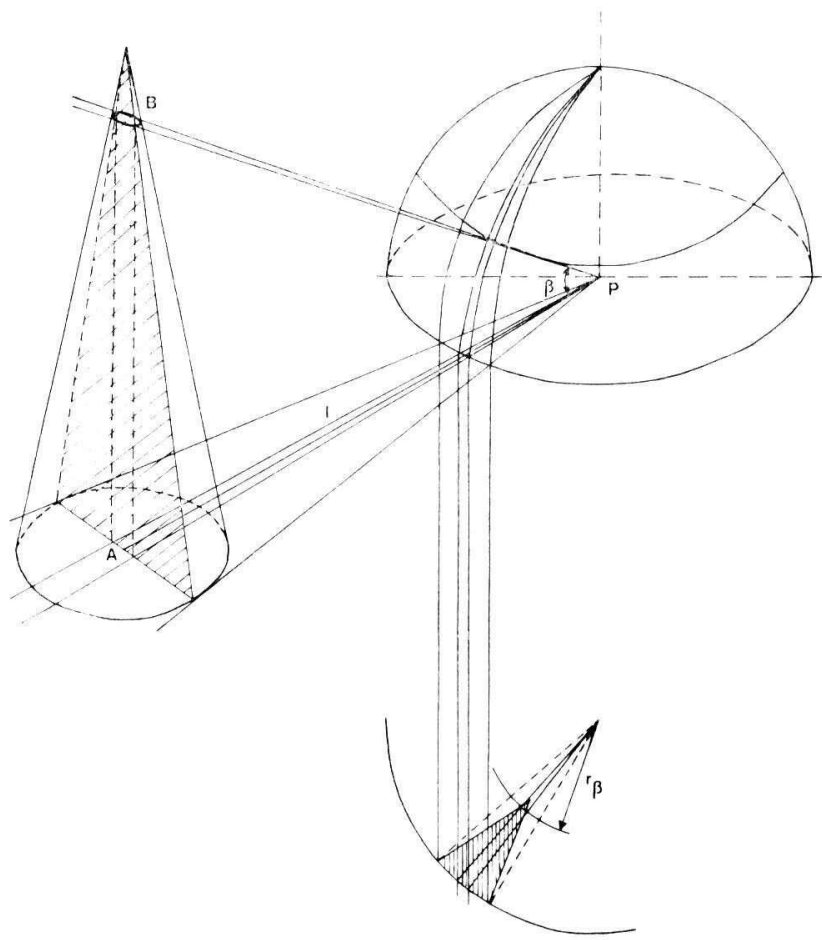

FIG. 2. - Représentation dans l'espace de la projection équidistante appliquée au cas de la mesure des hauteurs et diamètres sur photographies hémisphériques

FIG. 2. - Diagramm in the space of the equidistant projection in the case of hemispherical photographs showing how we calculate diameters and heights of trees

b) Cas d'un terrain en pente.

Lorsque le terrain est en pente, le système photographique doit être maintenu horizontal afin que les images des arbres soient toujours des droites convergeant vers le centre de la photographie et que les propriétés de la projection équidistante soient applicables.

On continuera à appeler $l$ la distance entre l'arbre et l'appareil photographique, mesurée parallèlement au terrain (figure 3). Le point $\mathrm{B}$ pour lequel on veut mesurer le diamètre est toujours situé à une hauteur angulaire $\beta$ au-dessus du plan de référence. Le point $\mathrm{A}$ dont la hauteur au-dessus du sol est $h_{0}$ (hauteur de l'appareil photographique au-dessus du sol) est maintenant situé à une hauteur angulaire $\beta^{\prime}$ au-dessus du plan horizontal.

La distance entre l'arbre et l'axe du dièdre (distance auparavant égale à $l$ ) est :

$$
l^{\prime}=l \cos \beta^{\prime} .
$$

La mesure du diamètre au point $\mathrm{B}$ n'est modifiée que par le fait que $l$ est remplacé par

$$
l \cos \beta^{\prime} .
$$




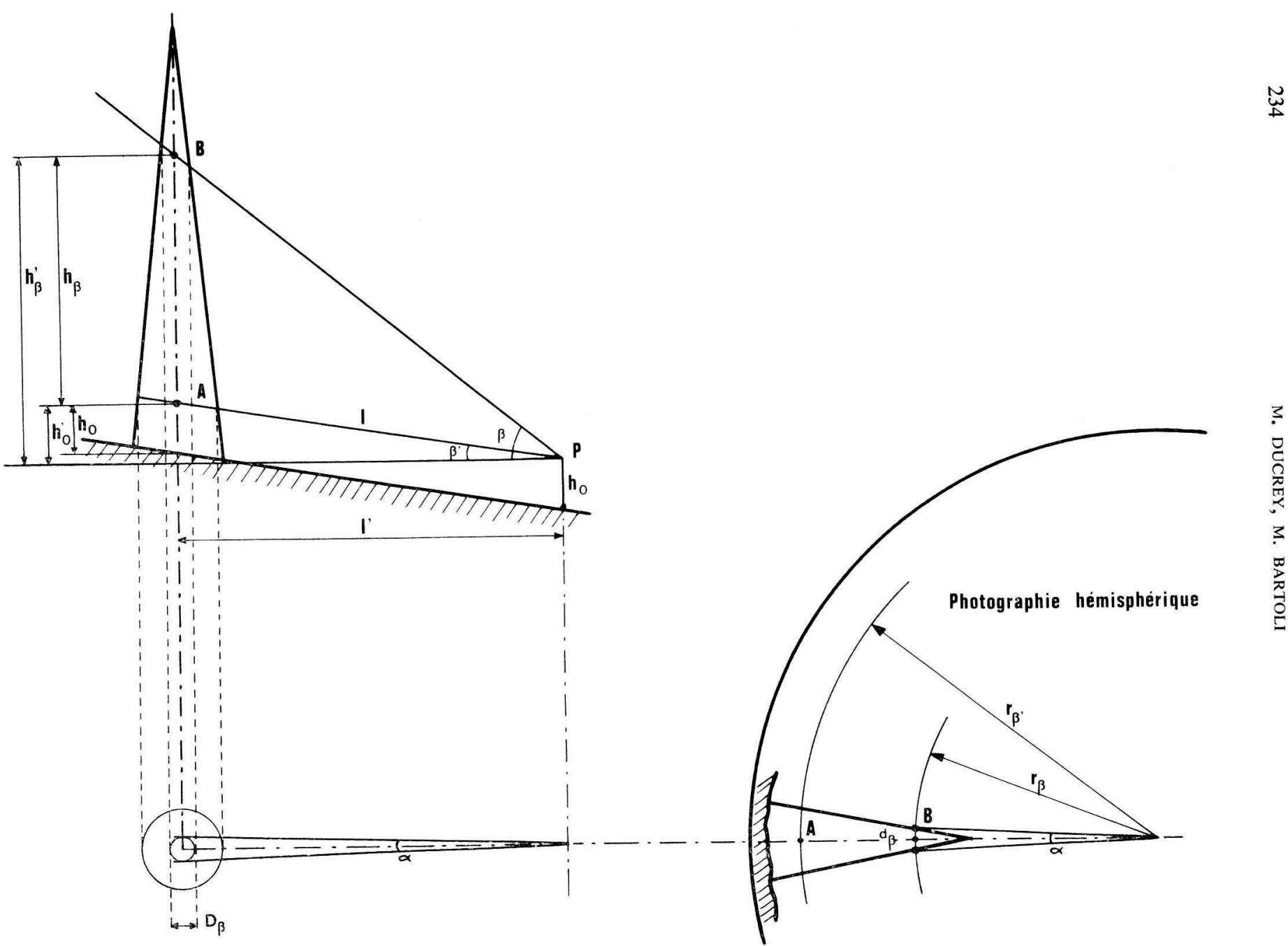

3
5
0
0
0
0
3
3
0
0
0
0
0

FIG. 3. - Mesure des hauteurs et diamètres sur photographies hémisphériques dans le cas d'un terrain en pente 
Le diamètre de l'arbre au point $\mathrm{B}$ correspondant à une hauteur angulaire $\beta$ au-dessus du plan de référence a pour valeur :

$$
\mathrm{D}_{\mathrm{B}}=l \cos \beta^{\prime} \frac{d_{\beta}}{r_{\beta}},
$$

quand la pente du terrain entre l'arbre et l'appareil est égale à $\beta$.

La hauteur du point de mesure $\mathrm{B}$ au-dessus du plan de référence est :

$$
h_{\beta}^{\prime}=l \cos \beta^{\prime} \operatorname{tg} \beta \text {. }
$$

La hauteur de mesure par rapport au pied de l'arbre est :

c'est-à-dire :

$$
\mathrm{H}_{\mathrm{B}}=h_{\mathrm{B}}^{\prime}-h_{0}^{\prime}+h_{0},
$$

$$
\mathrm{H}_{\beta}=l \cos \beta^{\prime} \operatorname{tg} \beta-l \sin \beta+h_{0} .
$$

Cette égalité est valable quel que soit $\beta^{\prime}$ si on considère $\beta^{\prime}$ positif quand l'arbre est au-dessus de l'appareil et inversement. Nous verrons que, dans la pratique, on ne peut mesurer $\beta^{\prime}$ sur la photographie que si $\beta^{\prime}$ est positif ou très faiblement négatif.

Nous venons de voir comment mesurer le diamètre d'un arbre à une hauteur donnée. Nous pouvons aussi mesurer la hauteur de n'importe quel repère particulièrement intéressant : hauteur du premier défaut, hauteur du houppier et même accroissement annuel en longueur pour certains arbres qui forment chaque année des verticilles facilement identifiables. Pour des raisons pratiques que nous évoquerons plus loin, il est difficile de mesurer avec précision la hauteur totale de l'arbre mais on peut, par contre, mesurer d'autres paramètres telles la dimension des houppiers ou la surface terrière des peuplements.

\section{2. - Mesure du diamètre des houppiers}

Le houppier d'un arbre est, en général, très irrégulier. La photographie hémisphérique ne permet d'en donner que le contour apparent suivant une section perpendiculaire à la direction d'observation.

Il s'agit toujours de mesurer, comme dans le cas du diamètre du tronc, une distance horizontale. Mais comme les dimensions du houppier sont grandes, on ne peut plus faire les approximations du cas précédent.

Nous étudierons le cas général d'un houppier asymétrique représenté par la figure 4 .

Le diamètre du houppier $\mathrm{D}$, calculé d'après la somme de ses deux rayons opposés $\mathrm{D}_{1}+\mathrm{D}_{2}$ s'écrit :

$$
\mathrm{D}=\mathrm{D}_{1}+\mathrm{D}_{2}=l \sin \alpha_{1}+l \sin \alpha_{2}
$$

En effet, les angles $\alpha_{1}$ et $\alpha_{2}$ sont trop grands pour que l'on puisse les utiliser à la place de leur sinus comme précédemment.

L'estimation de $\alpha_{1}$ et $\alpha_{2}$ sur le cliché photographique, en tenant compte du fait que la partie la plus large du houppier n'est pas obligatoirement à la même hauteur de chaque côté, est la suivante :

$$
\begin{aligned}
& d_{1}=r_{1} \sin \alpha_{1}, \\
& d_{2}=r_{2} \sin \alpha_{2} .
\end{aligned}
$$



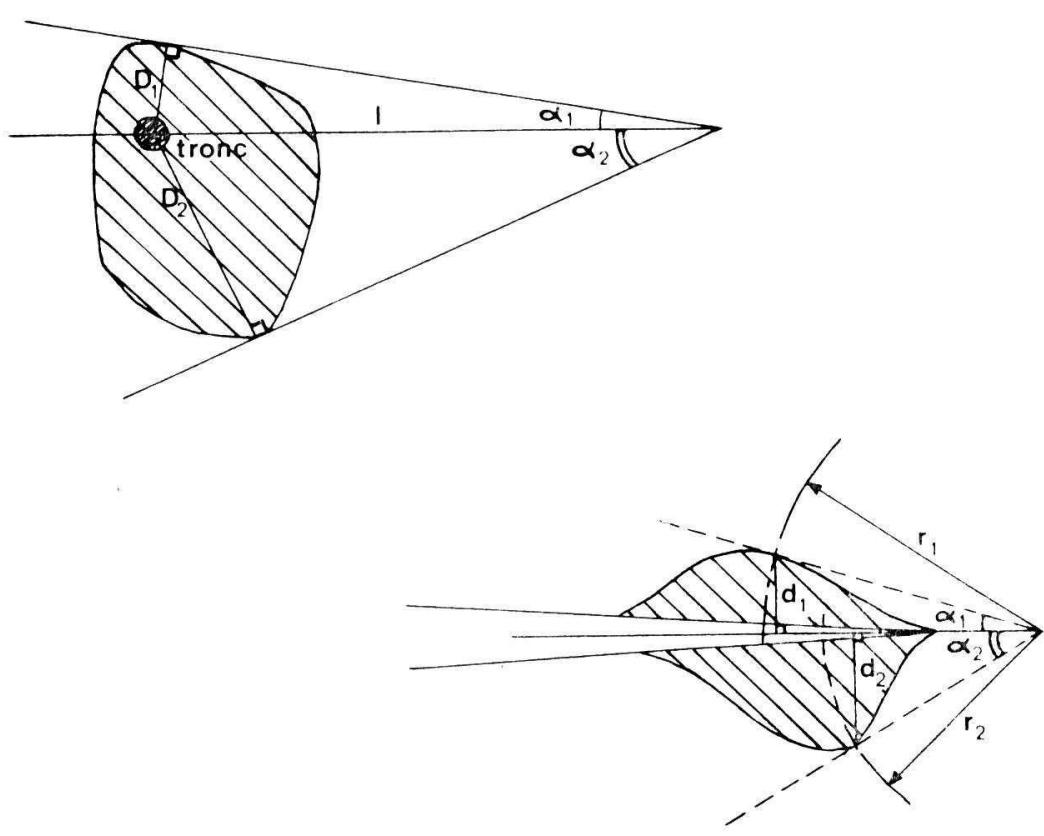

FIG. 4. - Mesure du diamètre d'un houppier asymétrique à l'aide des photographies hémisphériques FIG. 4. - Measurement of the diameter of an asymmetrical crown with hemispherical photographs

Il vient immédiatement :

$$
\mathrm{D}=l\left(\frac{d_{1}}{r_{1}}+\frac{d_{2}}{r_{2}}\right) .
$$

Dans le cas où la pente du terrain entre l'arbre et l'observateur est $\beta^{\prime}$ le diamètre du houppier est alors :

$$
\mathbf{D}=l \cos \beta^{\prime}\left(\frac{d_{1}}{r_{1}}+\frac{d_{2}}{r_{2}}\right) .
$$

L'estimation du diamètre moyen peut sembler insuffisante quand on mesure simplement deux rayons d'un houppier asymétrique. Nous verrons, dans le paragraphe concernant l'utilisation pratique de la méthode que l'opérateur est souvent amené à photographier le même arbre sous des angles de vue différents, ce qui donne une meilleure précision sur l'estimation du diamètre.

\section{3. - Mesure des surfaces terrières}

Il est bien sûr possible de mesurer la surface terrière d'un arbre particulier puisqu'on peut mesurer le diamètre à n'importe quelle hauteur, à $1,30 \mathrm{~m}$ en particulier. Ceci peut 
d'ailleurs être fait avec n'importe quel objectif photographique dont on connaît le rapport d'agrandissement (DECOURT, 1956).

Mais les photographies hémisphériques permettent en plus de mesurer les surfaces terrières par une véritable «méthode Bitterlich 》 telle qu'elle a été appliquée par BITTERLICH à l'aide de son relascope.

Le principe de la «méthode Bitterlich » est de calculer la surface terrière : G, en fonction du nombre d'arbres : N, qui sont vus, à partir d'un point donné et en faisant un tour d'horizon complet de balayage.

La relation est la suivante :

$$
\mathrm{G}=\mathrm{K} \cdot \mathrm{N},
$$

$\mathrm{K}$ étant une constante qui dépend de l'angle de balayage choisi : $\gamma$.

La démonstration mathématique de cette égalité (PARDE, 1961) montre que :

$$
\mathrm{G}=2500 \gamma^{2} \cdot \mathrm{N},
$$

si on exprime la surface terrière $G$ en $\mathrm{m}^{2} /$ ha et l'angle $\gamma$ en radians.

En ce qui concerne les photographies hémisphériques, il suffira de prendre le cliché à $1,30 \mathrm{~m}$ de hauteur et de compter à la périphérie du cliché, tous les arbres dont le diamètre sur photographie est supérieur à une longueur déterminée par l'angle de balayage et le rayon du cliché.

PARDÉ préconise d'avoir à compter toujours un nombre d'arbres compris entre 20 et 30 afin d'éliminer certaines erreurs. Ceci revient à choisir un angle de balayage adapté à la surface terrière probable du peuplement.

Le tableau 1 montre quelles sont les valeurs à donner à l'angle $\gamma$ pour compter un nombre d'arbres voisin de 25 quand on a une idée approximative de la surface terrière du peuplement.

$$
\text { TABLEAU } 1 \text { - TABLE } 1
$$

\begin{tabular}{|c|c|c|c|c|c|c|c|c|c|c|}
\hline $\begin{array}{l}\text { Surface terrière } G \text { en } \\
\mathrm{m}^{2} / \text { hectare } \ldots \ldots \ldots \ldots\end{array}$ & 5 & 10 & 15 & 20 & 25 & 30 & 35 & 40 & 45 & 50 \\
\hline $\begin{array}{l}\text { Angle de balayage } \gamma \\
\text { en radians (pour } \\
\mathrm{N}=25) \ldots \ldots \ldots \ldots \ldots\end{array}$ & 0,0089 & 0,0126 & 0,0155 & 0,0179 & 0,0200 & 0,0219 & 0,0237 & 0,0253 & 0,0268 & 0,0283 \\
\hline
\end{tabular}

Valeurs del'angle de balayage $\gamma$ pour le «tour d'horizon Bitterlich 》 en fonction de la surface terrière quand on a $N=25$.

Il suffira ensuite de multiplier l'angle de balayage choisi, par le rayon le plus grand de la photographie pour déterminer la longueur du segment à déplacer à la périphérie de la photographie.

Dans le cas d'un terrain en pente, la photographie devra être prise parallèlement à la pente, de telle manière que l'on puisse toujours mesurer le diamètre à 1,30 $\mathrm{m}$ à la périphérie du cliché. 
Nous n'en dirons pas plus au sujet des surfaces terrières car nous n'avons pas véritablement expérimenté cette méthode.

\section{II. - DESCRIPTION ET UTILISATION DE L'APPAREIL}

Nous indiquerons, dans ce chapitre, quelques conseils très importants pour l'utilisation du dendromètre et nous préciserons les opérations pratiques de dépouillement au laboratoire des photographies hémisphériques.

\section{1. - Description du dendromètre}

L'objectif photographique utilisé pour nos mesures est un « fish-eye » NIKKOR, de 7,5 mm de focale et de 1/5,6 d'ouverture maximale. Cet objectif qui utilise le principe de la projection équidistante, peut être placé sur les boîtiers Nikon ou Nikkormat.

Nous n'utilisons pas de films spéciaux. Les seules contraintes sont les suivantes :

- la luminosité générale qui varie suivant le type de temps et la densité du peuplement forestier,

- la différence de luminosité entre le zénith et l'horizon ce qui peut poser quelques problèmes si on veut que la base des troncs apparaisse nettement sans que la partie supérieure ne soit «mangée » par un excès de luminosité,

- la vitesse du vent qui fait remuer la cime des arbres et qui nécessite d'utiliser des vitesses assez grandes,

- la finesse du grain de la pellicule afin de donner une très bonne définition des contours de l'image.

Mais ce sont là des problèmes que doit résoudre n'importe quel photographe amateur!

En vue de faciliter la mise en station du dendromètre, nous avons imaginé de visser sur l'objectif une couronne plane de $20 \mathrm{~cm}$ de diamètre environ. Cette couronne matérialise le plan de référence de l'objectif photographique et doit donc être installée horizontalement.

Elle est munie :

- d'un niveau à bulle qui permet de maintenir la couronne horizontalement quand l'appareil est monté sur un trépied, et qui, par conséquent, assure la verticalité de l'axe optique de l'objectif,

- d'une boussole qui sert à orienter la photographie,

- et d'un reticule formé par deux fils d'acier qui se croisent orthogonalement dans l'axe optique de l'objectif et dont l'image sur le cliché permet de déterminer le centre exact de la photographie.

\section{2. - Utilisation pratique du dendromètre}

L'emploi de l'appareil sur le terrain est extrêmement simple. Il est mis en station horizontalement à une distance appropriée de l'arbre ou du groupe d'arbres concernés. Nous 
verrons au paragraphe suivant, qu'on a intérêt à avoir une distance comprise entre le tiers et la moitié de la hauteur des arbres étudiés.

En ce qui concerne la hauteur du dendromètre au-dessus du sol, l'opérateur a le choix entre deux possibilités :

Si le dendromètre est placé exactement à $1,30 \mathrm{~m}$ du sol, et si on est dans le cas du sol parfaitement horizontal, on peut utiliser les formules simplifiées correspondantes. Mais comme le sol n'est jamais parfaitement horizontal, on a intérêt à placer le dendromètre à une hauteur voisine de 1 mètre, de manière à ce que le niveau $1,30 \mathrm{~m}$ soit parfaitement identifiable sur les arbres en cause. Ce repère sert ensuite de référence pour le calcul du volume. Les hauteurs supérieures à $1,30 \mathrm{~m}$ et les diamètres correspondants sont mesurés sur la photographie. Il reste à mesurer au ruban le diamètre à $1,30 \mathrm{~m}$ ainsi que le diamètre à $0,50 \mathrm{~m}$.

En résumé, la série d'opérations préliminaires à effectuer est la suivante :

1) Choisir la hauteur de station du dendromètre (chose faite en principe, une fois pour toutes).

2) Déterminer l'emplacement du dendromètre en suivant les indications sur la distance et en évitant que les arbres à mesurer ne cachent ou ne soient cachés en partie par d'autres arbres.

3) Identifier les arbres à mesurer en leur donnant un numéro et en matérialisant la hauteur à 1,30 $\mathrm{m}$ de manière à ce que ces deux indications soient lisibles sur le cliché.

4) Mesurer au ruban le diamètre à $1,30 \mathrm{~m}$ (à titre de vérification) et le diamètre à $0,50 \mathrm{~m}$.

5) Mesurer avec beaucoup de précision $( \pm 0,05 \mathrm{~m})$ la distance entre l'axe du dendromètre et le centre de l'arbre; cette distance est à prendre parallèlement au sol.

6) Mesurer la hauteur totale de l'arbre (avec un dendromètre Blume Leiss) si cette indication est utile, car cette mesure est difficile à faire sur la photographie, surtout si le dendromètre est trop rapproché de l'arbre.

Les premiers essais faits sur le terrain montrent que l'ensemble de ces opérations prend un temps assez court. La mise en station de l'appareil, la prise du cliché et la mesure des distances à 4 arbres, nécessitent un temps de 4 minutes. Il faut ajouter une durée de $1 \mathrm{mi}-$ nute par arbre pour les mesures de hauteurs et de diamètres à $1,30 \mathrm{~m}$ et $0,50 \mathrm{~m}$ quand elles sont nécessaires.

En définitive, il faut, à une équipe de 2 personnes, 8 minutes de travail sur le terrain pour recueillir les données nécessaires au cubage de 4 arbres.

\section{3. - Exploitation des photographies}

Les négatifs sont projetés sur un écran translucide de manière à permettre de travailler à l'arrière de l'écran. Le grain de l'écran devra être aussi fin que possible pour avoir une meilleure définition de l'image. Un rapport d'agrandissement voisin de 50 semble suffisant pour permettre la mesure des diamètres apparents avec une bonne précision. Ceux-ci sont mesurés au pied à coulisse au dixième de millimètre. Les hauteurs angulaires corres- 
pondant aux niveaux de mesure des diamètres sont repérées sur une réglette graduée en degrés pour un agrandissement donné.

Quand on mesure 7 diamètres à des hauteurs différentes pour cuber un arbre par billons, il faut, d'après nos essais, deux minutes de travail par arbre pour une équipe de deux personnes. Cette opération est donc très rapide et elle a l'avantage de pouvoir être faite en série.

\section{4. - Utilisation pour les calculs de volume}

Le calcul du volume se fait très simplement à partir des circonférences $C_{0,50}$ et $C_{1,30}$ et des diamètres $D_{\beta}$ mesurés pour différentes hauteurs. Le choix de ces hauteurs est important. Si on se réfère à la méthode de cubage par le dendromètre BARR et STROUD, on voit que les visées sont faites de telle manière que le sinus de l'angle de visée augmente à chaque fois d'une valeur constante. Dans ces conditions et en tenant compte du fait que ce dendromètre permet de viser au maximum à $45^{\circ}$, les billons de la base de l'arbre sont plus courts que ceux du sommet.

C'est ce que nous avons fait dans le tableau 2 en «découpant » un arbre de $23 \mathrm{~m}$ de hauteur en billons mesurant de 2 à $5 \mathrm{~m}$.

TABLEAU 2 - TABLE 2

Valeurs des hauteurs angulaires (en degrés) à utiliser pour avoir des billons de taille optimum, en fonction de la distance entre l'arbre (hauteur $23 \mathrm{~m}$ ) et le dendromètre.

\begin{tabular}{|c|c|c|c|c|c|c|c|c|}
\hline & & \multicolumn{7}{|c|}{$\begin{array}{l}\text { Hauteur du niveau de mesure par rapport } \\
\text { au plan de référence (hauteurs en mètres) }\end{array}$} \\
\hline & & 2 & 4 & 7 & 10 & 14 & 18 & 23 \\
\hline & 3 & 34 & 53 & 67 & 73 & 78 & 81 & 83 \\
\hline Distance & 5 & 22 & 39 & 55 & 64 & 70 & 75 & 78 \\
\hline entre & 7 & 16 & 30 & 45 & 55 & 64 & 69 & 73 \\
\hline l'arbre & 10 & 11 & 22 & 35 & 45 & 55 & 61 & 67 \\
\hline $\begin{array}{c}\text { et le } \\
\text { dendromètre }\end{array}$ & 15 & 8 & 14 & 25 & 34 & 43 & 50 & 57 \\
\hline (en mètres) & 20 & 6 & 11 & 19 & 27 & 35 & 42 & 49 \\
\hline
\end{tabular}

Nous avons ensuite déterminé la valeur des angles $\beta$ à utiliser, pour pouvoir « découper » l'arbre toujours de la même manière, quelle que soit la distance entre l'arbre et l'appareil photographique. 
Ces résultats sont mis en évidence dans les schémas de la figure 5.
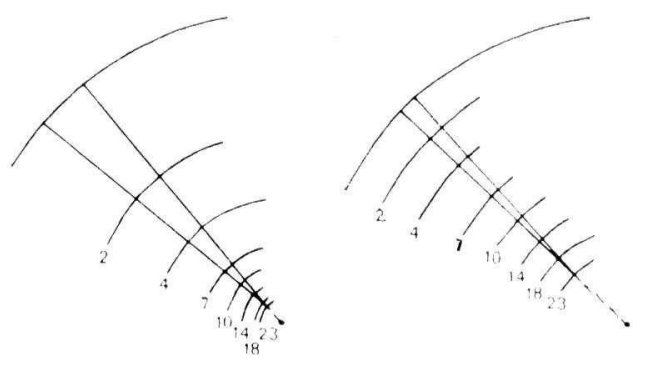

Image d'un arbre

$$
\begin{aligned}
& \text { a } 3 \mathrm{~m} \text { de } \\
& 1 \text { apparesl photos }
\end{aligned}
$$

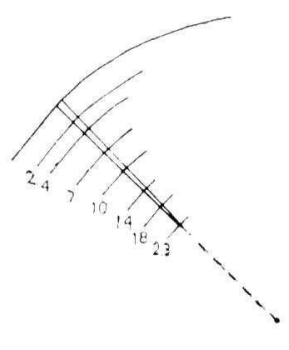

a $20 \mathrm{~m}$

FIG. 5. - Schéma montrant l'image, sur une photographie hémisphérique, d'un arbre haut de $23 \mathrm{~m}$ $\left(\mathrm{D}_{1,30}=0,50 \mathrm{~m}\right)$ pour différentes positions du « dendromètre » par rapport à l'arbre

FIG. 5. - Diagramm indicating how an hemispherical photograph changes when the camera is at different distances from the tree (tree height $=23 \mathrm{~m} ; \mathrm{Dbh}=\mathrm{D}_{1.30}=0,50 \mathrm{~m}$ )

Lorsque le dendromètre est à $3 \mathrm{~m}$ de l'arbre haut de $23 \mathrm{~m}$, l'image de l'arbre est assez grande et on peut donc avoir une bonne précision sur la mesure de $d_{\beta}$ jusqu'à 7 à $10 \mathrm{~m}$ de hauteur, mais au-delà l'image est très « tassée » sur elle-même et il devient hasardeux de mesurer $d_{\beta}$.

A l'opposé, quand le dendromètre est à $20 \mathrm{~m}$ de l'arbre, les niveaux de mesure sont très régulièrement espacés jusqu'au sommet. Cependant l'arbre apparaît alors trop petit sur la photographie et la mesure des diamètres apparents devient trop imprécise.

Par contre, pour une distance de l'ordre de $10 \mathrm{~m}$, l'arbre n'est ni trop déformé par la projection, ni trop éloigné du dendromètre.

Ces résultats sont confirmés par nos propres essais et l'on peut dire en guise de résumé que le dendromètre doit être situé à une distance variant entre le tiers et la moitié de la hauteur des arbres à mesurer. Dans ces conditions, le sommet de l'arbre atteint sur la photographie des hauteurs angulaires voisines de 60-70 degrés.

\section{III. - PREMIERS RÉSULTATS}

Les premiers travaux ont été réalisés dans des peupleraies en raison de la facilité qu'on y trouve pour prendre les photographies. Il faut, en effet, que le contour des arbres soit parfaitement visible depuis le bas du tronc jusqu'au sommet, et ce n'est pas toujours chose facile à réaliser.

Ceci implique que les troncs soient suffisamment éclairés comme c'est le cas des peuplements feuillus pendant l'hiver. C'est pourquoi, sauf cas de peuplements très éclairés ou récemment éclaircis, la méthode ne s'appliquera que très rarement aux peuplements résineux. Mais, c'est aussi une difficulté que l'on rencontre avec les dendromètres utilisant des systèmes de mesure optiques (Blume-Leiss, Barr et Stroud, pentaprisme, Bitterlich...). 
Au cours d'une première expérience, les circonférences de 10 peupliers ont été mesurées par grimpage pour des hauteurs variant de mètre en mètre de 2 à $12 \mathrm{~m}$. Les diamètres correspondants ont été mesurés sur photographies hémisphériques. Les observations portent au total sur 73 diamètres. La figure 6 montre que l'écart entre les diamètres réels et les diamètres mesurés sur photographies est en moyenne très proche de zéro. Un test de Wilcoxon nous a prouvé qu'il n'y avait pas de biais systématique entre les deux types de mesure et c'est un résultat particulièrement intéressant pour le calcul des volumes. En effet, les erreurs individuelles auront tendance à se compenser et à réduire l'erreur finale sur le volume.

Des observations faites sur les diamètres à $1,30 \mathrm{~m}$ pour un plus grand nombre d'arbres : 110 peupliers Robusta et 69 peupliers I 214 montrent (figure 7) une légère sous-estimation du diamètre par les photographies hémisphériques (de l'ordre de 2 à 3 p. 100 en moyenne). Cela est dû au fait que le diamètre à $1,30 \mathrm{~m}$ est difficile à mesurer sur la photographie quand on place le dendromètre exactement à $1,30 \mathrm{~m}$ de hauteur comme nous l'avons fait dans l'expérience en question.

Nous en tirons deux conclusions immédiates :

- il vaut mieux placer le dendromètre nettement au-dessous de $1,30 \mathrm{~m}(1 \mathrm{~m}$ par exemple) et matérialiser très clairement le niveau $1,30 \mathrm{~m}$ sur l'arbre,

— il est préférable de « doubler » la mesure à $1,30 \mathrm{~m}$ par une mesure de la circonférence à $1,30 \mathrm{~m}$ avec un ruban.

En ce qui concerne le volume, nous n'avons pas encore de données complètes permettant de comparer le volume réel d'un peuplier et son volume d'après photographie. A titre indicatif, nous pouvons dire que le tarif de cubage par photographies hémisphériques, actuellement en cours de construction pour le peuplier I 214, donne des résultats conformes à celui publié par l'AFOCEL (1969) et établi de manière classique par grimpage.

Nous sommes cependant en mesure de prévoir l'erreur sur le volume lorsque l'on connaît les erreurs sur la distance $l$ et sur le diamètre apparent $d_{\beta}$.

Nous avons pour cela calculé le volume d'un arbre en supposant que les mesures de $l$ et de $d_{\beta}$ sont exactes. Ensuite, nous avons calculé l'erreur relative commise sur le volume total en simulant des erreurs sur $l$ et $d_{\beta}$.

L'erreur relative sur le volume a été calculée pour des erreurs absolues de $\pm 10 \mathrm{~cm}$ et $\pm 20 \mathrm{~cm}$ sur la longueur $l$, et $\mathrm{de} \pm 0,2 \mathrm{~mm}$, $\pm 0,5 \mathrm{~mm}$ et $\pm 1 \mathrm{~mm}$ pour les diamètres apparents $d_{\beta}$ comme le montre la figure 8 .

L'erreur sur la distance $l$ est une erreur qui reste la même dans la gamme de distances considérée, puisqu'elle dépend essentiellement de la manière d'apprécier la position du centre de l'arbre. On peut l'estimer à $\pm 10 \mathrm{~cm}$ si l'on opère sans précaution particulière et à $\pm 5 \mathrm{~cm}$ si la mesure est faite avec soin. C'est donc cette dernière valeur que nous pouvons retenir.

L'erreur sur le diamètre apparent $d_{\beta}$ est celle qui est faite au moment de la lecture sur l'écran de projection. Avec l'écran utilisé et pour une photographie dont le diamètre atteint 1 mètre en projection, nous avons constaté des erreurs absolues variant de 0,2 à $0,5 \mathrm{~mm}$ pour des diamètres apparents de 1 à $30 \mathrm{~mm}$. Cette erreur est relativement élevée et, ainsi que le montre la figure 8 , elle a un poids important dans l'erreur sur le volume. 


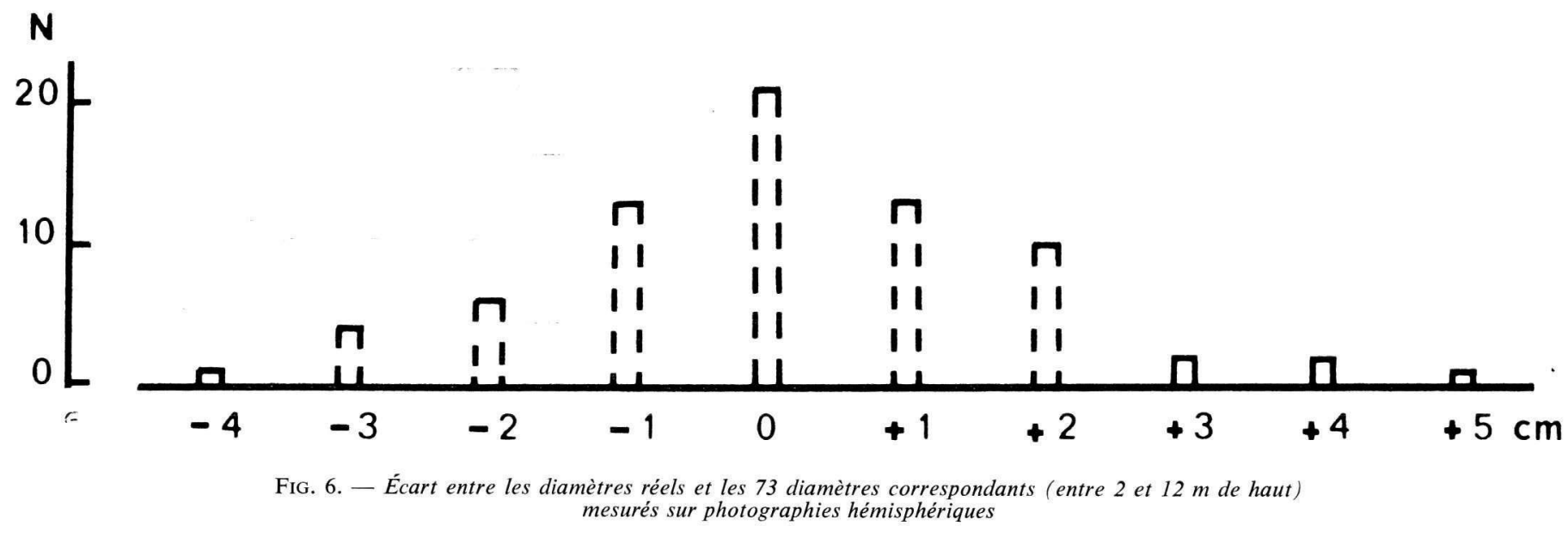

FIG. 6. - Difference between actual diameters and diameters measured on hemispherical photographs (73 trees with heights ranging from $\mathbf{2} \mathrm{m}$ to $\mathbf{1 2} \mathrm{m}$ ) 
N

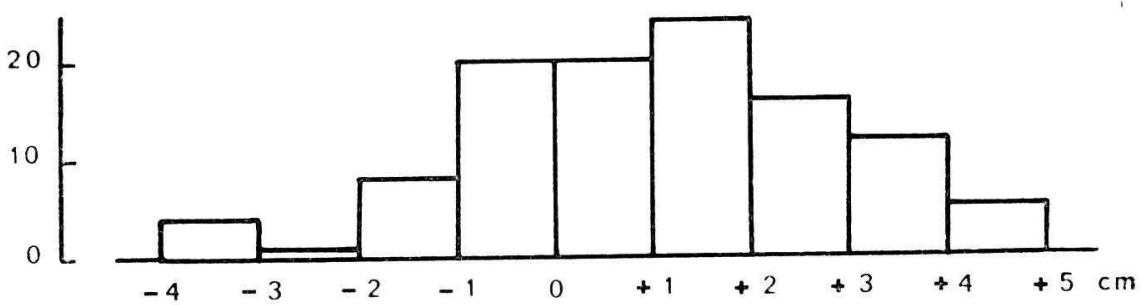

D 1,30 - DPHOT pour 110 Robusta

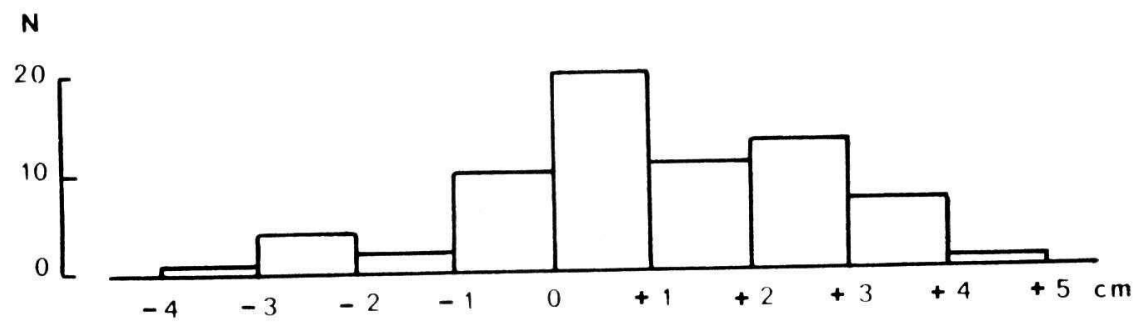

D 1,30-DPHOT pour 69 I214

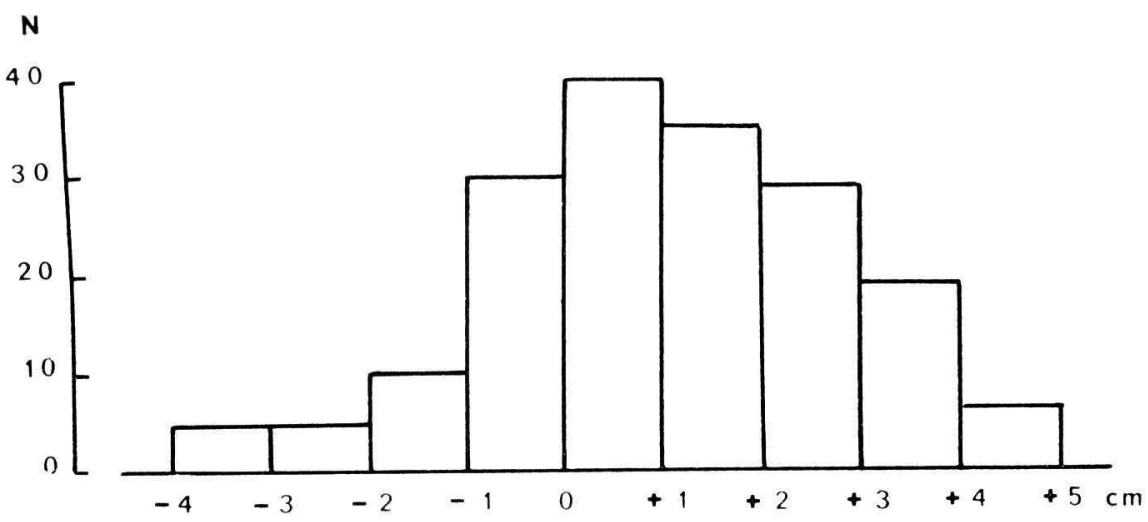

D 1,30 - DPHOT pour les 179 arbres mesurés

FIG. 7. - Écart entre les diamètres réels à $1,30 \mathrm{~m}\left(\mathrm{D}_{1,30}\right)$ et les diamètres à $1,30 \mathrm{~m}$ mesurés sur photographies hémisphériques (DPHOT) pour deux clônes de peupliers

FIG. 7. - Difference between actual breast height diameters $\left(\mathrm{D}_{1,30}\right)$

and breast height diameters measured on hemispherical photographs (DPHOT) for two poplar clones 


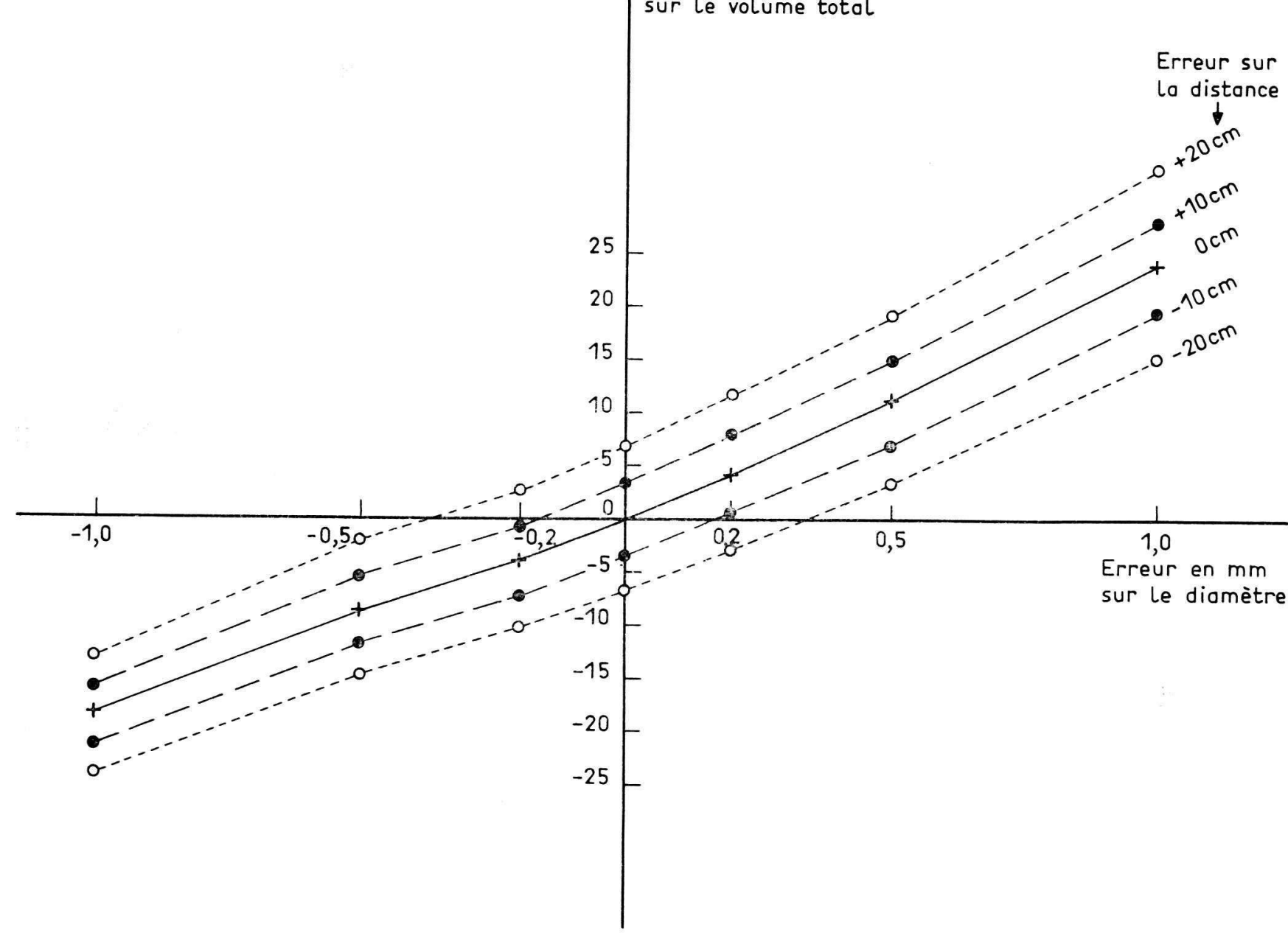

Erreur sur distance

FIG. 8. - Erreur relative sur le volume calculée en fonction de l'erreur absolue sur le diamètre "photographie 》 $\left(\mathrm{d}_{\beta}\right)$ pour différentes erreurs absolues sur la distance réelle arbre-« dendromètre » (1)

FIG. 8. - Relative error on the volume for different absolute errors on the distance tree-camera (1) by taking account of the absolute errors on the diameter measured on the photograph $\left(\mathrm{d}_{\beta}\right)$ 
Elle peut être diminuée très sensiblement (jusqu'à 0,1 ou $0,2 \mathrm{~mm}$ ) si on utilise un écran qui diffuse peu la lumière. En effet, l'écran translucide sur lequel nous avons travaillé facilite la tâche de l'opérateur (car il peut se placer derrière l'écran) mais il crée un phénomène de pénombre qui empêche de discerner avec précision les contours extérieurs de l'arbre.

A la lumière de ce qui vient d'être dit, nous pensons qu'au stade final d'utilisation de cette méthode, l'erreur relative sur le volume d'un arbre sera voisine de 5 p. 100.

\section{IV. - AVANTAGES ET INCONVÉNIENTS DE LA MÉTHODE}

La mise en œuvre de cette méthode nécessite un certain matériel :

- un appareil photographique équipé d'un objectif « fish-eye » et d'un trépied;

- un projecteur à diapositives possédant une bonne optique sans distorsion;

- un écran translucide de bonne qualité afin d'éviter les phénomènes de pénombre.

L'achat de ce matériel peut sembler onéreux mais on remarquera qu'il n'est absolument pas spécifique de ce genre de travail.

Un second critère très important est la précision de la méthode. Nous avons vu au paragraphe précédent que les mesures de diamètres ne sont pas biaisées, ce qui est très important, et que l'on peut escompter une précision de 5 p. 100 pour le calcul du volume d'un arbre.

Que représente cette précision par rapport aux méthodes classiques de cubage?

Si on mesure au réel les volumes, l'erreur faite reste de l'ordre de 2 à 3 p. 100.

Si on connaît la hauteur et 5 diamètres dont 1 à hauteur d'homme et 4 grimpés, l'erreur possible varie entre 0 et 5 p. 100. C'est dans cette gamme d'erreurs que se situent les dendromètres permettant de mesurer les diamètres à différentes hauteurs.

Si on connaît seulement la hauteur et le diamètre à hauteur d'homme l'erreur pourra atteindre 20 p. 100. C'est ce qui se passe quand on utilise une formule de cubage traditionnelle où seul intervient le coefficient de forme. L'erreur est ramenée entre 5 et 8 p. 100 si on emploie une formule de cubage complexe et exacte.

Avec les photographies hémisphériques, l'erreur doit ne pas excéder 5 p. 100 si on veut que la technique soit compétitive avec les dendromètres permettant des mesures analogues.

On veillera donc à ce que toutes les précautions dont nous avons longuement parlé aux paragraphes précédents, soient parfaitement respectées.

Un troisième critère intéressant est la facilité de mise en œuvre et la rapidité de la méthode. Il y a déjà un très grand avantage dans le fait que le travail sur le terrain est réduit au minimum. Nous avons vu qu'une équipe de deux personnes peut en 8 minutes recueillir les données concernant 4 arbres (sur une même photographie) y compris les diamètres à $0,50 \mathrm{~m}$ et à $1,30 \mathrm{~m}$ et la hauteur totale. A titre de comparaison, il faut à peu près 20 minutes pour " cuber» un seul arbre avec le dendromètre Barr et Stroud. Le dépouillement des photographies au bureau est un travail supplémentaire par rapport aux autres méthodes mais il n'excède pas 2 minutes par arbre.

La réduction du temps de travail sur le terrain est un avantage primordial. En effet, 
sur un plan pratique, le temps de déplacement des équipes est limité et on a intérêt à recueillir le maximum d'information pendant le minimum de temps même si le travail au bureau s'en trouve légèrement accru.

Disons au passage que les équipes peuvent travailler à peu près tout le temps en dehors des moments de pluie ou de vent pendant lesquels il n'est plus possible de photographier.

Les utilisations « annexes » des photographies hémisphériques en matière de dendrométrie sont le dernier point sur lequel nous insistons.

Les mesures de diamètre de houppier peuvent être utilisées par exemple dans des tarifs de cubage pour les inventaires par photographies aériennes.

Les photographies hémisphériques en peuplement forestier sont de véritables « tours d'horizon Bitterlich ». Nous avons d'ailleurs vu au $1^{\mathrm{er}}$ paragraphe comment elles permettent de calculer la surface terrière d'un peuplement. Elles pourront donc servir de base pour la réalisation d'inventaires statistiques. On voit dès maintenant la rapidité avec laquelle de tels inventaires pourraient être réalisés, et aussi toutes les possibilités qui s'ouvrent tant dans la modification de la densité d'échantillonnage que celle de la surface des placettes élémentaires.

Énumérons au passage d'autres observations pouvant être faites sur photographies hémisphériques :

- mesure de la hauteur du $1^{\mathrm{er}}$ défaut,

- mesure de la hauteur du houppier,

- mesure du diamètre des branches,

- détermination de l'accroissement annuel en longueur dans certains cas précis.

La photographie hémisphérique nous apparaît, en fin de compte, comme une image objective, précise et à «l'échelle » d'un peuplement à un instant donné. C'est, enfin, un document particulièrement riche d'information pour celui qui, à partir de séries de photographies, veut suivre l'évolution de la structure du peuplement tout au long de sa vie.

Reçu pour publication en octobre 1974.

SUMMARY

UTILIZATION OF HEMISPHERICAL PHOTOGRAPHS FOR

VOLUME MEASUREMENT OF STANDING TREES

A new method for measuring volume of standing trees using a camera with a $180^{\circ}$ fish-eye lens is proposed. Heights and diameters are calculated from measurements made on photographic enlargements. Accuracy of this method (5 p. 100 on the total volume of a tree) is fairly similar to that of the classical methods in which we add volumes of successive logs (Smalian or Huber method). 


\section{ZUSAMMENFASSUNG}

DIE VERWENDUNG VON WEITWINKEL-

\section{AUFNAHMEN ZUR MASSENERMITTLUNG AN STEHENDEN B̈̈UMEN}

Vom Verfasser wird eine neue Methode der Massenermittlung an stehenden Bäumen vorgeschlagen. An Vergrösserungen von photographischen Aufnahmen $(24 \times 36)$ mit einem Weitwinkelobjektiv « fish-eye » (Offnungswinkel $180^{\circ}$ ) werden Hohen und Durchmesser gemessen und sektionsweise die Massen berechnet. Die Genauigkeit der Methode (ca $5 \%$ der Schaftholzmasse m.R.) entspricht annähernd jener, welche bei Stehendmessung mit üblichen Geräten erreicht wird.

\section{RÉFÉRENCES BIBLIOGRAPHIQUES}

Barneoud C., Bonduelle P. - Volume sur pied et production de peupleraies « I 214 » réparties dans l'Est de la France. Afocel 1969, p. 173-208.

Decourt N., 1956. - Utilisation de la photographie pour mesurer les surfaces terrières. R.F.F. Vol. 8, $\mathrm{n}^{0} 7$, p. 505-507.

DUCReY M., 1970. - Utilisation des photographies hémisphériques pour l'évaluation de la densité du couvert forestier et pour le calcul de la perméabilité au rayonnement solaire. Publication interne Station Sylviculture C.N.R.F., $\mathrm{n}^{\circ} 70-4$.

DuCREY M., 1971. - Contribution des photographies hémisphériques à l'étude du microclimat lumineux en forêt. Publication interne Station Sylviculture C.N.R.F., $\mathrm{n}^{\circ} 71-1$.

PARdé J. - Dendrométrie. Éditions École Nationale des Eaux et Forêts. Nancy 1961. 